\title{
Physical Activity and BMI in a Nationally Representative Sample of Children and Adolescents
}

\author{
Arlene E. Chung, MD, MHA ${ }^{1}$, Asheley Cockrell Skinner, PhD ${ }^{1}$, Michael J. Steiner, MD ${ }^{1}$, and \\ Eliana M. Perrin, MD, MPH ${ }^{1}$ \\ ${ }^{1}$ University of North Carolina at Chapel Hill, Chapel Hill, NC, USA
}

\section{Abstract}

Objective-To examine objectively measured physical activity levels by age, sex, and BMI for children and adolescents in a nationally representative sample.

\begin{abstract}
Methods-Data were from the 2003-2004 and 2005-2006 National Health and Nutrition Examination Surveys, which included physical activity assessment by accelerometer and measured height and weight. The authors calculated minutes of moderate and vigorous activity.

Results-Boys were more active than girls, and activity levels were lower at older ages. Younger children met daily recommendations for physical activity, whereas older children, especially girls, did not. Typically, weight status was inversely related to activity, though differences were less apparent among boys. Underweight children were not always more active than heavier peers.
\end{abstract}

\section{Keywords}

obesity; physical activity; children and adolescents; age; body mass index; BMI

\section{Introduction}

Obesity remains one of the most pressing public health issues facing children and adolescents in the United States. Recent data from the 2007-2008 National Health and Nutrition Examination Survey (NHANES) indicate that approximately 32\% of 2- to 19-yearold children in the United States are overweight, with $17 \%$ being obese and $12 \%$ very obese. ${ }^{1}$ Considerable research links lack of physical activity to childhood obesity and associated comorbidities. ${ }^{2-5}$ Physical activity for children and adolescents has also been shown to have beneficial effects on body weight, ${ }^{6}$ and children who are active are more likely to remain active during adolescence and into adulthood, ${ }^{7}$ making physical activity in childhood even more important. For all these reasons and others, the 2008 Physical Activity Guidelines for Americans recommends that children and adolescents spend at least 60 minutes in moderate to vigorous physical activity daily. ${ }^{8}$ Despite the health benefits of physical activity and these recommendations, very few children and adolescents meet these standards, ${ }^{9}$ and little is known about the relationship of weight status to measured physical activity.

\section{(C) The Author(s) 2012}

Corresponding Author: Arlene E. Chung, 5034 Old Clinic Building, CB\#7110, University of North Carolina at Chapel Hill, Chapel Hill, NC 27599-7110, USA, arlene_chung@med.unc.edu.

Reprints and permission: agepub.com/journalsPermissions.nav

Declaration of Conflicting Interests

The authors declared no potential conflicts of interest with respect to the research, authorship, and/or publication of this article. 
Physicians caring for overweight and obese children need better information about the activity levels of these children. Clear understanding of differences in physical activity levels by weight status and by age would improve the ability to tailor recommendations throughout childhood. Although a recent study examined physical activity by weight and race/ethnicity, this report does not provide age-specific breakdowns by weight and sex, which may reveal different patterns and be critical to clinical recommendations for physical activity. ${ }^{10}$

Social desirability and the difficulty of estimating the frequency and duration of physical activity ${ }^{11,12}$ often leads to overreporting of activity when based on self-report. Therefore, objective measurements of physical activity are important to estimate levels of activity accurately throughout childhood. A number of studies have validated the use of accelerometers, which are devices that can detect directional movements, to determine physical activity levels in children and adolescents. ${ }^{13-15}$ Prior research with accelerometers found sex differences in physical activity levels, with boys being more active than girls, and that those differences increased during adolescence. ${ }^{9,16}$ Additionally, there are some data to suggest that overweight children are generally less active than healthy-weight children, but this came from a small US sample. ${ }^{17}$ Body mass index (BMI) status may influence whether children meet the daily recommendations for physical activity, but further investigation is warranted in this era of increased rates of obesity.

Although prior studies have examined age and sex differences in objectively measured activity, more research is needed to examine how BMI status influences whether children meet the daily recommendations for physical activity. To our knowledge, little is known about physical activity in very obese children and underweight children because those who are underweight may also not meet recommendations for activity and therefore may not be obtaining the cardiovascular benefits of exercise. Thus, the goals of this study are to examine levels of objectively measured physical activity in children aged 6 to 17 years old in a nationally representative sample by sex and BMI, to determine whether weight status influences whether children meet the daily recommendation of greater than 60 minutes of moderate to vigorous activity daily, to examine sustained physical activity, and to examine physical activity in underweight children and compare it with physical activity in their heavier peers.

\section{Methods}

\section{Data Source}

We used data from NHANES (2003-2004 and 2005-2006). NHANES is a stratified, multistage probability sample of the US population. NHANES is conducted throughout the year at 15 geographic sites selected annually. It includes data from a physical examination, laboratory testing, and a detailed in-home interview. The physical examination includes measured height and weight and 7 days of accelerometer-based monitoring of physical activity. ${ }^{18,19}$

\section{Weight Status}

Weight status was defined using BMI percentiles calculated from measured height and weight. We defined weight categories according to the recommended cut-points from the Centers for Disease Control as follows: <5th percentile, underweight; 5th to 85th percentile, healthy weight; >85th to 95th percentile, overweight; >95th to 99th percentile, obese. Also, $>99$ th percentile was used to determine very obese based on expert committee recommendations from the American Academy of Pediatrics. ${ }^{20}$ 


\section{Accelerometers}

NHANES used the Actigraph AM-7164 accelerometer (Actigraph, LLC; Ft Walton Beach, FL) to track uniaxial movement. Further details of the accelerometer protocol in NHANES are available. ${ }^{21}$ Accelerometers tracked the intensity of vertical movements, summed over 1-minute time intervals, and the intensity was recorded as "counts." The device was worn on the right hip on a fabric belt fitted for each participant. Because the accelerometer was not waterproof, it was not worn during water-based activities such as swimming or bathing. The participants were instructed to wear the accelerometers while awake for 7 consecutive days and remove them at bedtime nightly during the 7-day period.

\section{Sample}

We included all ambulatory children who were 6 to 17 years old $(\mathrm{N}=4718)$. After excluding those who did not have proper calibration of their accelerometers or who did not have at least 4 days or more of $\geq 10$ hours of accelerometer wear per day, ${ }^{22,23}$ our final analytic sample included 1560 girls and 1587 boys. All data were weighted to represent the US population.

\section{Interpretation of Accelerometer}

Data and Analyses-We used the National Cancer Institute's SAS program, as recommended by NCHS for NHANES analysis, to create analytic data sets for accelerometer data. ${ }^{24}$ This program corrects the per-minute accelerometer data that have invalid monitor readings by replacing it with the average of adjacent values.

Accelerometer counts are classified into a standard measure of physical activity called metabolic equivalent tasks (METs). A MET of 1 indicates the energy expended at rest during a given period of time. We aimed to create measures representing time spent in moderate or vigorous activity. There is currently no consensus for how to account for standard differences in energy expenditure between children and adults. Based on the most frequently used definitions in previous pediatric activity literature, we defined moderate physical activity as equivalent to 4 METs and vigorous physical activity as equivalent to 7 METs. ${ }^{25-27}$ A sensitivity analysis in children and adolescents comparing different cut-points for METs showed that Freedson's equation, which was used in this study, best adjusted for the higher energy expenditure in a broad range of ages in youth. ${ }^{28}$ For example, 4 METs would be expended by activities such as playing hopscotch, and 7 METs would be defined by activities such as running or jumping rope. There have also been validation studies to examine various MET cut-points in children and the cut-points used in our study. ${ }^{13,14}$

In terms of classifying accelerometer counts into METs, children of different ages appear to produce different accelerometer counts for similar levels of metabolic activity; thus, multiple equations have been developed that include age as a variable. Because of the broad age group of children examined in our study, we used an age-adjusted equation developed by Freedson and colleagues and published by Trost et al, ${ }^{14}$ where counts are defined as counts in a single minute, and age is age in years:

$$
\text { METs }=2.757+\left(0.0015 \times \text { Counts }-{ }^{1}\right)-(0.08957 \times \text { Age })-\left(0.000038 \times \text { Counts }-{ }^{1} \times \text { Age }\right) .28,29
$$

Using this equation, we calculated the counts per minute necessary to be considered moderate or vigorous activity based on the child's age. For each day, we calculated the total number of average minutes spent in moderate activity or vigorous activity and average minutes of sustained activity, defined as activity maintained for greater than or equal to 8 minutes of any 10-minute period based on the National Cancer Institute's code for sustained 
activity. ${ }^{24}$ Though the 2008 Physical Activity Guidelines for Americans does not recommend specific amounts of sustained activity, we were interested in how much sustained activity was performed as well. Finally, we created a child-level data set that combined the activity measures for the days the accelerometer was worn. Adherence to physical activity recommendations was also examined by determining the proportion of children in each age group by BMI status that met the daily recommendations for activity.

We also examined bivariate comparisons of mean number of minutes spent per day in moderate or vigorous activity, by weight status. We examined girls and boys separately and used age groups to determine differences across childhood. We tested for differences using adjusted Wald tests for continuous variables and the Pearson $F$-statistic with the Rao and Thomas correction for categorical variables. All analyses were adjusted according to NCHS recommendations and were performed using the svy commands in Stata 11.0 (StataCorp, College Station, TX). ${ }^{30,31}$

\section{Results}

The analytic sample included 3147 children, which was $67 \%$ of the eligible sample that participated in the accelerometer portion of NHANES. There were similar numbers of girls and boys, and there were no significant differences in age, race, or income by sex (Table 1). In terms of compliance with accelerometer wear, there were no significant differences across BMI categories for girls and boys overall, with the exception that underweight boys who were 9 to 11 years old had significantly more hours of monitor wear.

\section{Physical Activity Trends by Age Groups}

Overall, girls and boys of all ages who were underweight and of healthy weight spent more time in moderate and in vigorous physical activity than overweight, obese, or very obese children (Table 2). On average, underweight and healthy-weight children spent approximately 10 minutes in vigorous activity and 49 to 55 minutes in moderate activity per day. Generally, the amount of time spent in moderate or vigorous physical activity was less for those who were overweight and obese.

Healthy-weight girls of all age categories were more active in moderate and vigorous activity than their heavier peers. Underweight girls were more active than overweight or obese girls but generally spent less average minutes in activity than healthy-weight children. Younger age groups were more active than older age groups.

In boys, significant differences based on weight status were evident when ages were pooled with a stepwise increase in activity with decreasing weight status. There were only significant differences by BMI status in the 6- to 8-, 9- to 11-, and 15- to 17-year-old groups for vigorous activity and in the 15- to 17-year-old group for moderate activity. Healthyweight boys were more active than their heavier peers. Unlike girls, healthy-weight boys spent less time on average in vigorous activity than their underweight peers.

\section{Sustained Physical Activity by Age Group}

Table 3 presents mean minutes of sustained physical activity, which was defined as at least 8 minutes of any 10-minute period of moderate to vigorous activity. For most children, sustained activity did not occur on a daily basis; therefore, most of the average daily values were less than 8 minutes. Overall, girls and boys of all ages spent very little time in sustained physical activity, though healthier-weight children were more active than overweight or obese peers. In general, children spent more time in sustained moderate physical activity than sustained vigorous activity. 
For girls, healthy-weight children of all ages were more physically active in sustained activity than their heavier peers. Younger age groups did not spend much time performing sustained moderate or vigorous activities, but those in the 6- to 8-year-old groups were the most active. Adolescent girls were the least active, with 12- to 17-year-old girls spending only small amounts of time on average in sustained moderate activity (5 minutes or less) and even less time performing sustained vigorous activity ( 2 minutes or less) on average irrespective of BMI status.

Among boys of all ages, sustained mean moderate and vigorous activity was significantly different by weight status. All age groups, except those who were 15 to 17 years old, had significant differences in sustained vigorous activity across BMI status. The reverse was true for sustained moderate activity, with significant differences by weight only seen in the 15- to 17-year-old group. Younger boys again were generally more active than older boys, as was the trend in girls. On average, boys aged 15 to 17 years (excluding underweight boys) spent less than an average of 1.5 minutes per day in sustained vigorous activity and only a few minutes more on average in sustained moderate activity per day than sustained vigorous activity.

\section{Percentage Meeting}

Daily Recommendations-To examine whether US children meet the daily recommendations of 60 minutes of moderate to vigorous activity daily, we examined the percentage of children in each age category by age, sex, and BMI categories (Figures 1A and 1B). Among 6- to 8-year-old girls, more than $90 \%$ of healthy-weight and overweight girls met the recommendations, whereas approximately $70 \%$ of obese and very obese girls did so. For boys, regardless of BMI status, nearly all 6- to 8-year-olds came close to meeting the daily recommendations of 60 minutes of moderate to vigorous physical activity. In the 9to 11-year-old category, slightly more than half of underweight and healthy girls and boys met daily recommendations for activity, whereas girls and boys who were overweight and obese/very obese had much lower percentages that met the daily recommendations. This pattern was also seen in girls and boys in the 12- to 14- and 15- to 17-year-old groups but was more pronounced. Less than 5\% of 12- to 14-year-old girls that were healthy weight, overweight, or obese/very obese met the recommendations. Surprisingly, a higher percentage (43\%) of 12- to 14-year-old boys who were very obese met the recommendations than those in other BMI categories. In the 15- to 17-year-old category, there were almost no girls that met the recommendations, and only a small percentage of boys met the daily recommendations regardless of BMI.

\section{Discussion}

Overall, we found that BMI status was related to physical activity for both girls and boys through childhood and adolescence. As seen in previous research, boys are generally more active than girls, and activity levels are lower in older age groups. ${ }^{8,13}$ Younger children generally met the recommendation of 60 minutes of moderate or vigorous physical activity daily, whereas older children and adolescents did not.

Weight status was significantly related to activity, particularly for girls. Healthy-weight girls of all ages were more active than overweight, obese, and very obese girls. It is interesting to note that we found few differences in total average levels of activity by weight for boys except in the oldest age group (15-17 years). However, healthy-weight boys did engage in more vigorous activity than their heavier peers. Contrary to previous research, ${ }^{17}$ overweight/ obese children were not always less active than their healthy-weight peers-particularly overweight/obese boys. One reason for the sex differences in physical activity by weight status may be that certain athletic activities may favor larger boys and create a perceived 
advantage to being a heavier weight status among boys or higher self-efficacy. We cannot assert causality from the results of this study, but further research is needed to explore activity in overweight/obese boys and what factors may be influencing them to be more active. Also, many underweight girls and boys did not meet daily recommendations, so many of these children are not receiving the health benefits of physical activity, though there may be health reasons why this is the case for this subgroup, and further research is necessary.

Regardless of the reasons for sex differences, our findings suggest the need to focus on physical activity at a young age, particularly for girls. Development of early activity habits may help sustain them through childhood. However, when addressing obesity in older children and adolescents, physicians should be cognizant that activity differences among boys by weight status may not be substantial, and focus on other aspects, such as diet, may prove more beneficial.

There are a few important limitations to our study. First, accelerometers detect uniaxial movement, so activities such as upper-body exercises are not recorded as accurately as activities such as walking or running and may thus underestimate activity levels.

Additionally, accelerometer data are dependent on device wearing compliance. In this study, there were no significant differences in compliance in wearing the device by sex. However, these limitations must be contrasted with the significant limitations of other activity studies that rely on self-reported physical activity because this objectively measured populationlevel study provides valuable insight into the levels of activity in US children by weight status and sex. Second, interpreting accelerometer data in children remains an area of ongoing research. Although there have been a few studies to compare varying cut-points and to determine which pediatric energy expenditure equations are the best for estimating physical activity intensity, there is no consensus. But there have been studies to compare pediatric cut-points and energy expenditure equations that show that the equation of Freedson et al equation is valid. ${ }^{12-14,24-26}$ Third, this is a cross-sectional study, so we cannot comment on how physical activity changes for an individual child throughout childhood or determine if there is a causative link between obesity and lack of physical activity or what that direction of causation would be. However, the use of nationally representative data gives us an opportunity to extrapolate to the overall child and adolescent population.

As children get older, there are fewer opportunities for required physical activity in the school setting. Required physical activity in schools throughout childhood and adolescence may help more children meet activity recommendations, and further investigation into other opportunities to increase all types of activity are needed. Providing physical activity recommendations at well-child visits may be another avenue to help encourage children to be more active, especially in adolescence when activity levels drop precipitously. Additionally, further research is needed to examine physical activity in underweight children because there is little known about why these children are also not meeting recommendations.

\section{Conclusions}

Our study finds that activity levels vary by weight status and confirms that activity levels also decline as children age into adolescence. All children who do not have activity limitations should be encouraged to be physically active regardless of weight status. Decreased physical activity as children become adolescents may be an important factor in perpetuating obesity, and increasing physical activity should be emphasized as children become adolescents. Targeted interventions are needed to increase levels of physical activity 
in those who do not meet the recommended amount of daily physical activity, especially for girls and adolescents. In particular, interventions that help increase physical activity for girls throughout childhood and adolescence and overcome perceived and actual barriers to activity may help more of them meet recommendations.

\section{Acknowledgments}

ACS had full access to all the data in the study and takes responsibility for the integrity of the data and the accuracy of the data analysis. AEC is supported by a NIH/HRSA training grant and is a NRSA Primary Care Research Fellow (T32HP14001). ACS is supported by a NIH career development award (K12HD001441). EMP is supported by a NIH career development award (K23 HD051817).

Funding

The authors received no financial support for the research, authorship, and/or publication of this article.

\section{References}

1. Ogden CL, Carroll MD, Curtin LR, Lamb MM, Flegal KM. Prevalence of high body mass index in US children and adolescents, 2007-2008. JAMA. 2010; 303:242-249. [PubMed: 20071470]

2. Anderson PM, Butcher KE. Childhood obesity: trends and potential causes. Future Child. 2006; 16:19-45. [PubMed: 16532657]

3. Krishnamoorthy J, Hart C, Jajalian E. The epidemic of childhood obesity: review of research and implications for public policy. Soc Policy Rep. 2006; 20(2):3-17.

4. Daniels SR, Arnett DK, Eckel RH, et al. Overweight in children and adolescents: pathophysiology, consequences, prevention, and treatment. Circulation. 2005; 111:1999-2012. [PubMed: 15837955]

5. Koplan, JP.; Liverman, CT.; Kraak, VI. Preventing Childhood Obesity: Health in the Balance. Washington, DC: National Academies Press; 2005.

6. Strong WB, Malina RM, Blimkie CJ, et al. Evidence based physical activity for school-age youth. J Pediatr. 2005; 146:732-737. [PubMed: 15973308]

7. Malina RM. Tracking of physical activity and physical fitness across the lifespan. Res Q Exerc Sport. 1996; 67 (3, suppl):S48-S57. [PubMed: 8902908]

8. Services USDoHaH. [Accessed January 2, 2010.] Physical activity guidelines for Americans. 2008. http://www.health.gov/PAGuidelines

9. Troiano RP, Berrigan D, Dodd KW, Masse LC, Tilert T, McDowell M. Physical activity in the United States measured by accelerometer. Med Sci Sports Exerc. 2008; 40:181-188. [PubMed: 18091006]

10. Belcher BR, Berrigan D, Dodd KW, Emken BA, Chou CP, Spuijt-Metz D. Physical activity in US youth: impact of race/ethnicity, age, gender, and weight status [published online ahead of print April 16, 2010]. Med Sci Sports Exerc.

11. deLeeuw, E.; Borgers, N.; Smits, A. Pretesting questionnaires for children and adolescents. In: Presser, S.; Rothgeb, JM.; Couper, MP., et al., editors. Methods for Testing and Evaluating Survey Questionnaires. New York, NY: John Wiley and Sons; 2004. p. 409-429.

12. Sallis JF, Saelens BE. Assessment of physical activity by self-report: status, limitations, and future directions. Res Q Exerc Sport. 2000; 71(2, suppl):S1-S14. [PubMed: 10925819]

13. Puyau MR, Adolph AL, Vohra FA, Butte NF. Validation and calibration of physical activity monitors in children. Obes Res. 2002; 10:150-157. [PubMed: 11886937]

14. Trost SG, Way R, Okely AD. Predictive validity of three ActiGraph energy expenditure equations for children. Med Sci Sports Exerc. 2006; 38:380-387. [PubMed: 16531910]

15. Trost SG, Ward DS, Moorehead SM, Watson PD, Riner W, Burke JR. Validity of the computer science and applications (CSA) activity monitor in children. Med Sci Sports Exerc. 1998; 30:629_ 633. [PubMed: 9565947]

16. Trost SG, Pate RR, Sallis JF, et al. Age and gender differences in objectively measured physical activity in youth. Med Sci Sports Exerc. 2002; 34:350-355. [PubMed: 11828247] 
17. Trost SG, Kerr LM, Ward DS, Pate RR. Physical activity and determinants of physical activity in obese and non-obese children. Int J Obes Relat Metab Disord. 2001; 25:822-829. [PubMed: $11439296]$

18. Centers for Disease Control and Prevention NCHS. [Accessed Febrauary 20, 2009.] NHANES documentation, codebook, and frequencies MEC exam component: physical activity monitor examination data. http://www.cdc.gov/nchs/data/nhanes/nhanes_03_04.paxraw_c.pdf

19. Centers for Disease Control and Prevention NCHS. [Accessed May 1, 2009.] NHANES documentation, codebook, and frequencies MEC exam component: body measures examination data. http://cdc.gov/nchs/data/nhanes/nhanes_03_04/bmx_c.pdf

20. Barlow SE. Expert committee recommendations regarding the prevention, assessment, and treatment of child and adolescent overweight and obesity: summary report. Pediatrics. 2007; 120(suppl 4):S164-S192. [PubMed: 18055651]

21. Centers for Disease Control and Prevention NCHS. [Accessed May 1, 2009.] NHANES laboratory procedures manual. http://www.cdc.gov/nchs/data/nhanes/nhanes_03_04/lab_pm.pdf

22. Trost SG, Pate RR, Freedson PS, Sallis JF, Taylor WC. Using objective physical activity measures with youth: how many days of monitoring are needed? Med Sci Sports Exerc. 2000; 32:426-431. [PubMed: 10694127]

23. Mark AE, Janssen I. Influence of bouts of physical activity on overweight in youth. Am J Prev Med. 2009; 36:416-421. [PubMed: 19362696]

24. National Cancer Institute. [Accessed April 5, 2009.] Risk factor monitoring and methods: SAS programs for analyzing NHANES 2003-2004 accelerometer data. http://riskfactor.cancer.gov/tools/nhanes_pam

25. Treuth MS, Schmitz K, Catellier DJ, et al. Defining accelerometer thresholds for activity intensities in adolescent girls. Med Sci Sports Exerc. 2004; 36:1259-1266. [PubMed: 15235335]

26. Roemmich JN, Clark PA, Walter K, Patrie J, Weltman A, Rogol AD. Pubertal alterations in growth and body composition: V. Energy expenditure, adiposity, and fat distribution. Am J Physiol Endocrinol Metab. 2000; 279:E1426-E1436. [PubMed: 11093932]

27. Harrell JS, McMurray RG, Baggett CD, Pennell ML, Pearce PF, Bangdiwala SI. Energy costs of physical activities in children and adolescents. Med Sci Sports Exerc. 2005; 37:329-336. [PubMed: 15692331]

28. Sirard JR, Heitzler CD, Lytle LA. Youth accelerometer cutoffs for moderate-to-vigorous physical activity: a sensitivity analysis. Med Sci Sports Exerc. 2009; 41:159.

29. Freedson P, Pober D, Janz KF. Calibration of accelerometer output for children. Med Sci Sports Exerc. 2005; 37(11, suppl):S523-S530. [PubMed: 16294115]

30. Centers for Disease Control and Prevention NCHS. [Accessed May 1, 2009.] NHANES analytic guidelines. Jun. 2004 http://www.cdc.gov/nchs/data/nhanes/nhanes_general_guidelines_june_04.pdf

31. Centers for Disease Control and Prevention NCHS. [Accessed May 1, 2009.] NHANES analytic and reporting guidelines. 2005. http://cdc.gov/nchs/data/nhanes/nhanes_03_04/nhanes_analytic_guidelines_dec_2005.pdf 


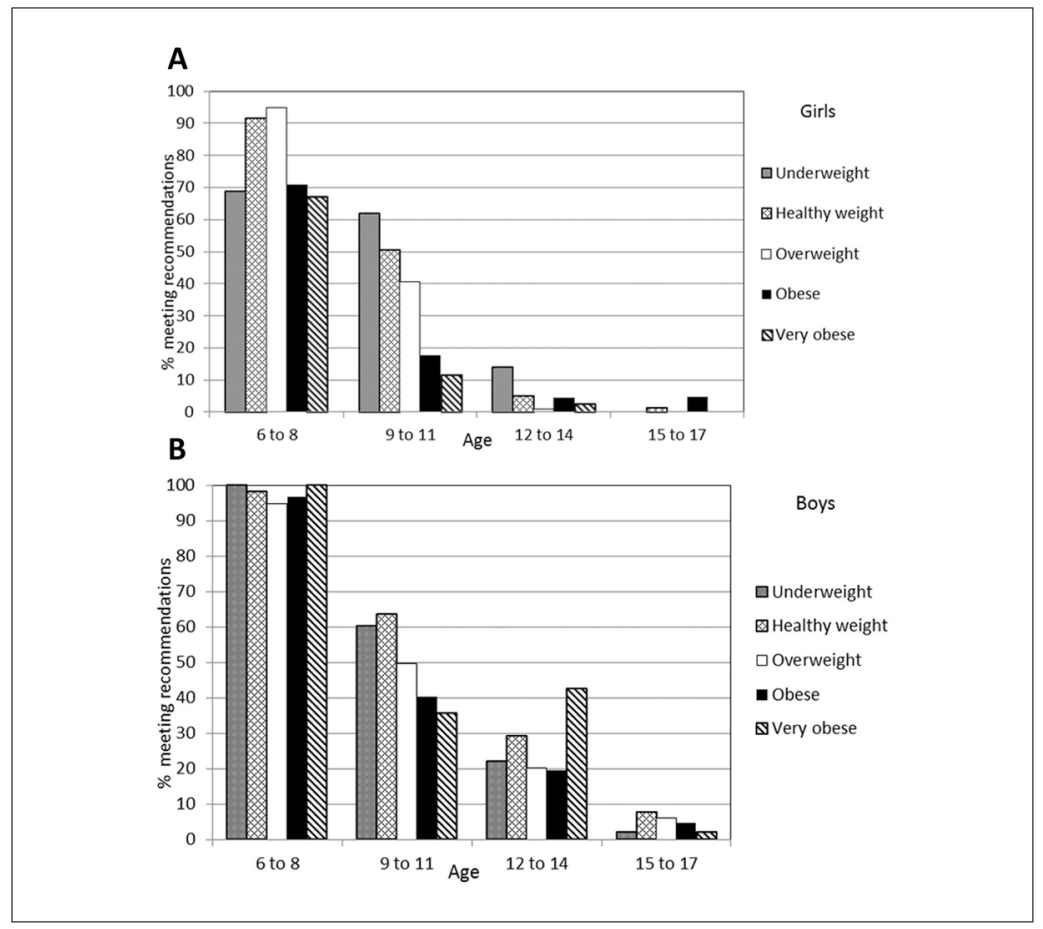

Figure 1.

Percentage meeting daily recommendations of 60 minutes of moderate to vigorous exercise, by age and BMI for girls (A) and boys (B) 
Table 1

Weighted Sample Characteristics

\begin{tabular}{|c|c|c|}
\hline & Girls $(n=1560)$, Percentage & Boys $(n=1587)$, Percentage \\
\hline \multicolumn{3}{|l|}{ Age in years } \\
\hline $6-8$ & 26.0 & 24.8 \\
\hline $9-11$ & 25.8 & 27.4 \\
\hline $12-14$ & 25.5 & 25.8 \\
\hline $15-17$ & 22.6 & 22.0 \\
\hline \multicolumn{3}{|l|}{ Race/Ethnicity } \\
\hline White & 60.0 & 60.0 \\
\hline Black & 14.2 & 15.3 \\
\hline Hispanic & 18.0 & 17.9 \\
\hline Other & 7.9 & 6.7 \\
\hline \multicolumn{3}{|l|}{ Income } \\
\hline$<100 \%$ FPL & 19.1 & 18.4 \\
\hline $100 \%-199 \%$ FPL & 21.6 & 21.5 \\
\hline $200 \%-299 \%$ FPL & 16.5 & 19.6 \\
\hline $300 \%-399 \%$ FPL & 17.3 & 13.1 \\
\hline $400 \%-499 \%$ FPL & 10.0 & 11.2 \\
\hline$>500 \% \mathrm{FPL}$ & 15.7 & 16.2 \\
\hline \multicolumn{3}{|l|}{ Weight category } \\
\hline Very obese & 3.3 & 3.4 \\
\hline Obese & 11.7 & 12.0 \\
\hline Overweight & 15.9 & 17.0 \\
\hline Healthy weight & 65.5 & 64.1 \\
\hline Underweight & 3.6 & 3.6 \\
\hline
\end{tabular}

Abbreviation: FPL, federal poverty level. 


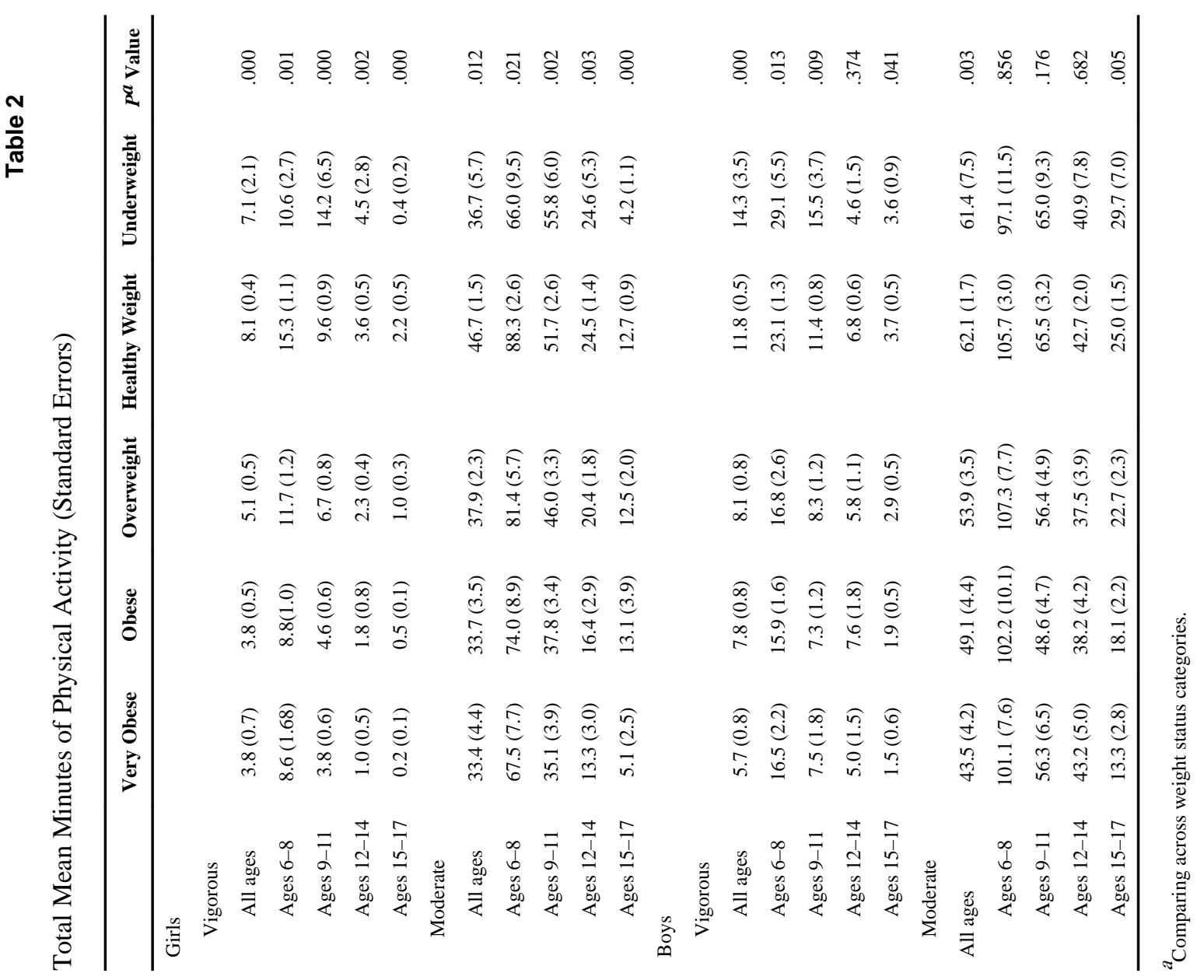


